\title{
A force-feedback exoskeleton for upper-limb rehabilitation in virtual reality
}

\author{
Antonio Frisoli*a ${ }^{*}$ Fabio Salsedo ${ }^{a}$, Massimo Bergamasco ${ }^{a}$, Bruno Rossi $^{\mathrm{b}}$ and Maria C. Carboncini ${ }^{\mathrm{b}}$ \\ ${ }^{a}$ PERCRO, Scuola Superiore Sant'Anna, Pisa, Italy; ${ }^{b}$ Neurorehabilitation Unit, Department of Neurosciences, University of Pisa, Italy
}

(Received 1 October 2008; final version received 1 April 2009)

\begin{abstract}
This paper presents the design and the clinical validation of an upper-limb force-feedback exoskeleton, the L-EXOS, for robotic-assisted rehabilitation in virtual reality (VR). The L-EXOS is a five degrees of freedom exoskeleton with a wearable structure and anthropomorphic workspace that can cover the full range of motion of human arm. A specific VR application focused on the reaching task was developed and evaluated on a group of eight post-stroke patients, to assess the efficacy of the system for the rehabilitation of upper limb. The evaluation showed a significant reduction of the performance error in the reaching task (paired $t$-test, $p<0.02$ ).
\end{abstract}

Keywords: exoskeleton; rehabilitation robotics; virtual reality

\section{Introduction}

Several research studies have recently focused both on the development of novel robotic interfaces and on the use of virtual reality (VR) technologies for neurorehabilitation. The former may overcome some of the major limitations manual-assisted movement training suffers from, i.e. lack of repeatability, lack of objective estimation of rehabilitation progress and the high dependence on specialised personnel availability. Thorough and constant exercise has revealed itself essential to produce a significant therapy outcome (Diller 2000). On the other hand, VR-based rehabilitation protocols may significantly improve the quality of rehabilitation by offering strong functional motivations to the patient who can therefore be more attentive to the movement to be performed. On the other hand, several studies (e.g. Jack et al. 2001) have demonstrated positive effects of VR on rehabilitation, which enhances cognitive and executive functions of stroke patients (Cardoso et al. 2006) by allowing them to receive enhanced feedback on the outcome of the rehabilitation tasks he/she is performing. Moreover, VR can provide an even more stimulating videogame-like rehabilitation environment when integrated with force feedback devices, thus enhancing the quality of the rehabilitation (Stewart et al. 2006).

Several arm rehabilitation robotic devices, both Cartesian and exoskeleton-based, have been developed in the last 10 years. Some examples include MIT Manus (Krebs et al. 1998; Fasoli et al. 2003), Assisted Rehabilitation and Measurement (ARM) guide (Reinkensmeyer et al. 2000), Mirror Image Movement Enabler (MIME) (Lum et al. 2002) and one-degree of freedom (DoF) and
two-DoF devices developed at Saga University (Kiguchi et al. 2001, 2003). A recent survey (Prange et al. 2006) outlines that robotic-aided therapy allows a higher level of improvement of motor control if compared with conventional therapy. Nevertheless, no consistent influence on functional abilities has yet been found.

Exoskeleton robots have recently raised the interest of the robotic rehabilitation research community. Exoskeletons are robotic systems designed to work linked with parts (or the whole) of the human body, as shown in Figure 1. In general, robots are designed for a defined workspace where they perform specific tasks autonomously (Avizzano and Bergamasco 1999). The design of exoskeleton systems stems from opposite motivations that intend the robotic structure to always maintain contact with the human operator's limb. Such a condition is required for several applications that include the use of master robotic arms for teleoperation, active orthoses and rehabilitation (Bergamasco 1996).

The strict correspondence of the exoskeleton workspace with the human limb's workspace defines constraints for the kinematics and range of joint motions of the exoskeleton robotic structure. Another design constraint is represented by the simultaneous presence of the limb volume and the robotic structure, i.e. due to the physical continuity of the body, the mechanical structure of the exoskeleton cannot occupy the same limb's volume and, consequently, it is usually shaped in order to wrap around the limb itself. Experiments on exoskeletons have been performed at the Jet Propulsion Laboratory (JPL) during 1970s (Jau, 1988). At Sarcos, Nahvi et al. (1998) developed a master

*Corresponding author. Email: a.frisoli@sssup.it 

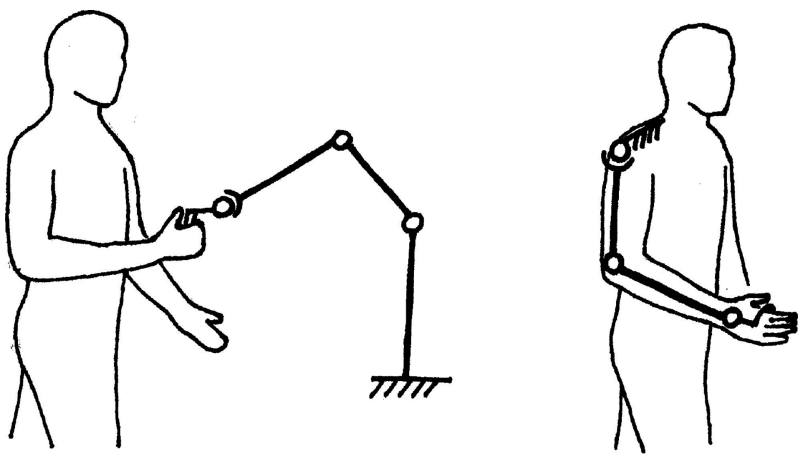

Figure 1. A comparison between the kinematic schemes of a classical manipulator and an exoskeleton system.

arm integrating also grasping capabilities for the hand used for the remote control of a robotic arm, while at PERCRO (Perceptual Robotics laboratory) the authors have developed arm exoskeletons for interaction with virtual environments since 1994 (Bergamasco 1996; Bergamasco et al. 1994; Frisoli et al. 2005). Exoskeletons can be suitably employed in robotic-assisted rehabilitation. The ARMin device (Nef and Riener, 2005; Riener et al. 2005) developed at ETH (Zurich Polytechnic University), Switzerland is a rehabilitation device that can provide three active DoFs for shoulder and one active DoF for elbow actuation. The Salford exoskeleton (Tsagarakis and Caldwell 2003), based on pneumatic muscle actuators (pMA), provides an excellent power over weight ratio and was used in physiotherapy and training, while Carignan et al. (2008) developed an exoskeleton system for physical therapy of shoulder, involving scapula motion. Gupta and OMalley (2006) presented the design of a haptic exoskeleton for training and rehabilitation, while Perry et al. (2007) designed a seven-DOF powered cable-driven arm exoskeleton for neurorehabilitation. Two exoskeleton-based systems were developed at Saga University, Japan. The older one (Kiguchi et al. 2001) is a one-DoF interface for human elbow motion, where angular position and impedance of the robot are tuned relying on biological signals used to interpret the human subject's intention, while the latest neuro-fuzzy-controlled device (Kiguchi et al. 2003) is a two-DoF interface used to assist human shoulder joint movement.

In this paper we present the design of an upperlimb force-feedback exoskeleton, the L-Exos, for roboticassisted rehabilitation in VR. The L-Exos is a five-DoF exoskeleton with a wearable structure and anthropomorphic workspace that can cover the full range of motion of a human arm. The system was validated on a group of eight post-stroke patients through a robotic-assisted therapy conducted in VR. The results indicate that exoskeleton systems can suitably be employed for upper-limb rehabilitation, since significant improvements were achieved in terms of quantitative analysis of the performance error of patients during the execution of the reaching task in VR.

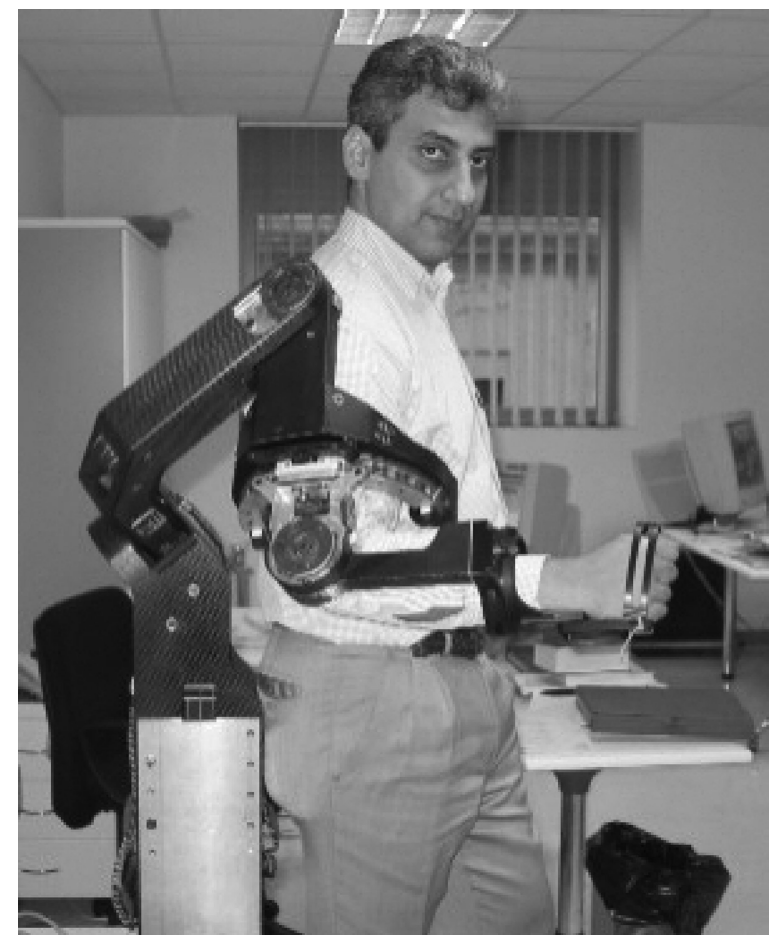

Figure 2. The PERCRO L-Exos device worn by a user.

The rest of this paper is organised as follows. In section 2 we present the design and the main features of the L-EXOS system. In section 3 we give an overview of the developed VR experimental set-up employed in this study for the validation of the exoskeleton in upper-limb rehabilitation, while in section 4 we present the results of the clinical evaluation.

\section{The L-EXOS system}

L-Exos (light exoskeleton) (Salsedo et al. 2002) is a forcefeedback exoskeleton for the human arm. The L-Exos has been designed as a wearable interface, capable of providing a controllable force at the centre of user's right-hand palm, oriented along any direction of the space, through a handle that can be grasped by the user. A button placed on the handle allows to perform basic selection operations in the virtual environment. For right-hand-impaired patients with grasping disabilities, the button can be remotely operated by the physiotherapist or by the patient's left hand (see Figure 2).

\subsection{Kinematics}

L-Exos is characterised by a serial kinematics consisting of five rotational joints (see Figure 3). The first four DoFs are both actuated and sensorised, while the fifth DoF is sensorised only with a potentiometer. The first three rotational joints are incident and mutually orthogonal (two by two) 


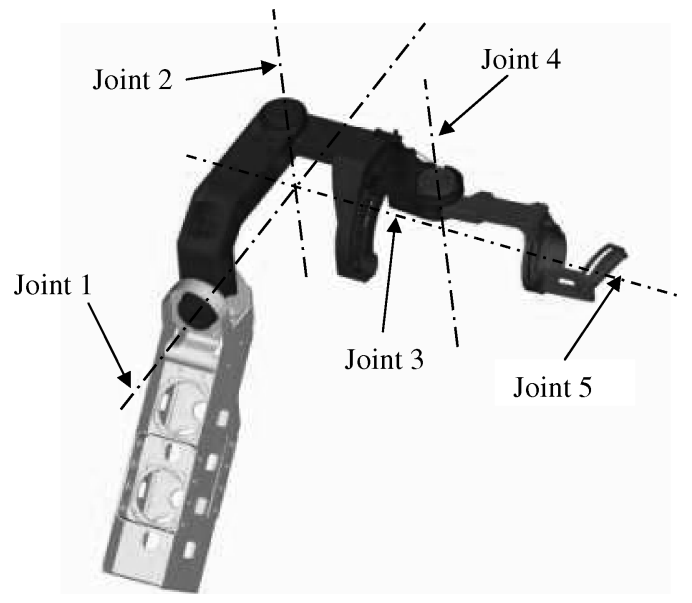

Figure 3. The general kinematics of the L-Exos.

in order to emulate the kinematics of a spherical joint with the same centre of rotation of the human shoulder, which is supposed to be fixed in space. The target workspace for the shoulder joint was assumed to be a spherical sector.

The orientation of the first joint was optimised in order to maximise the workspace of the shoulder joint by avoiding singularities and interferences between the mechanical links and the operator, resulting in a skewed position of the first link with respect to the horizontal and vertical plane. Moreover, the third joint was assumed to be coincident with the ideal axis of the upper arm, while the fourth and fifth joints were assumed to be coincident with the elbow joint and the forearm, respectively in order to allow the pronosupination of the wrist. The workspace of the elbow coincides with the angle of rotation of the fourth joint. Assuming a zero position for the condition of alignment between the forearm and the arm, the range of motion achieved by the L-Exos spans approximately from $2.5^{\circ}$ to $105^{\circ}$. The wrist has only one non-actuated DoF and its range of motion is of $180^{\circ}$. More precisely, it is of $\pm 90^{\circ}$ measured when the hand palm is aligned with arm and forearm in the zero position. Denavit-Hartenberg parameters for the L-Exos system are reported in Table 1 . The angles $\psi$ and $\phi$ parameters are set to $25^{\circ}$ and $40^{\circ}$ respectively. Moreover, $L_{1}$ and $L_{2}$ correspond to the lengths of the forearm and the arm respectively, which are both set to a value of $0.31 \mathrm{~m}$.

Table 1. Denavit-Hartenberg parameters for the L-Exos system.

\begin{tabular}{ccccc}
\hline $.7 \mathbf{i}$ & $\theta_{i}$ & $\alpha_{i}$ & $a_{i}$ & $d_{i}$ \\
\hline .81 & $\frac{\pi}{2}$ & $\frac{\pi}{2}$ & 0 & 0 \\
.72 & $\frac{\pi}{2}-\psi$ & $\frac{\pi}{2}$ & 0 & 0 \\
.83 & $\pi-\phi$ & $\frac{\pi}{2}$ & 0 & $-L_{1}$ \\
.74 & $\frac{\pi}{2}-\phi$ & $\frac{\pi}{2}$ & 0 & 0 \\
.84 & 0 & 0 & 0 & $L_{2}$ \\
\hline
\end{tabular}

\subsection{Mechanical design guidelines}

In order to improve the transparency of use of the device, a set of guidelines were adopted for the mechanical design.

All the motors of the exoskeleton were located at the fixed frame (link 0). For each actuated DoF, the torque is delivered from the motor to the corresponding joint by means of steel cables and a reduction gear integrated at each joint axis, as can be seen from the diagram of the transmission of axis 2 in Figure 4. Such an arrangement allows us to reduce the masses of the moving parts, by reducing the mass of the motors (about $40 \%$ of the overall mass of the exoskeleton) and the additional mass of the structural parts, to be reinforced in order to sustain the weight of heavier motors. The inertia perceived by the user at the palm is consequently reduced. For the same reasons electric actuators offering good torque to weight and torque to volume ratio were selected. To achieve a higher stiffness of the device at the end effector, reduction gears with a low reduction ratio were located at the joint axes, thus to allow the reduction of the tendon tension, length and diameter. The reduction of the tendon diameter led to a consequent saving of mass and volume of all the mechanical parts of the transmission system (pulleys, axles etc).

A general scheme of the adopted tendon transmission for the first three joints is shown in Figure 5, where the symbols $r_{i m}$ and $r_{i j}$ indicate the radii of the motor and joint pulley at joint $j$ of transmission $i$ respectively. From Figure 5 it is clear that the tendon transmission introduces a linear coupling between the joint displacements and this is reflected in the overall speed reduction ratio from motor to joint displacements. In fact, if we denote the motor and joint speed values by $\dot{q}_{m i}$ and $\dot{q}_{i}$, respectively, the speed reduction ratio introduced by the tendon transmission and the reduction gears is described by a linear relation

$$
\dot{\mathbf{q}}_{\mathbf{m}}=T G \dot{\mathbf{q}}
$$

where the matrices $T$ and $G$ in Equation (1), representing the tendon and gear reduction ratios respectively, are given as

$$
\left[\begin{array}{c}
\dot{q}_{m 1} \\
\dot{q}_{m 2} \\
\dot{q}_{m 3} \\
\dot{q}_{m 4}
\end{array}\right]=\left(\begin{array}{cccc}
t_{11} & 0 & 0 & 0 \\
t_{21} & t_{22} & 0 & 0 \\
t_{31} & t_{32} & t_{33} & 0 \\
t_{41} & t_{42} & t_{43} & t_{44}
\end{array}\right)\left(\begin{array}{cccc}
g_{11} & 0 & 0 & 0 \\
0 & g_{22} & 0 & 0 \\
0 & 0 & g_{33} & 0 \\
0 & 0 & 0 & g_{44}
\end{array}\right)\left[\begin{array}{c}
\dot{q}_{1} \\
\dot{q}_{2} \\
\dot{q}_{3} \\
\dot{q}_{4}
\end{array}\right] .
$$

The values of the reduction ratios $t_{i j}$ and $g_{i j}$ are given in Table 2. The lower triangular form of matrix $T$ is due to the linear coupling between the joints introduced by the tendon transmission, while the diagonal form of matrix $G$ is due to the presence of speed reducers on all joints, except the third one $\left(g_{33}=1\right)$. The reduction gears on joints 1 and 2 have a gear ratio of 6 , while on joint 4 there is a gear ratio 


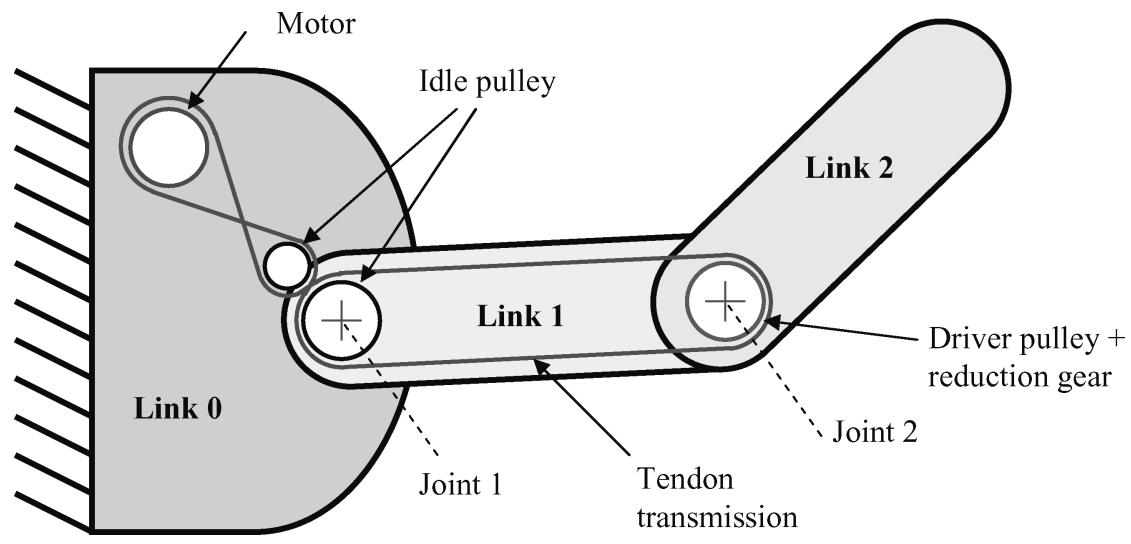

Figure 4. Actuation diagram for joint 2.

of 4 . The terms $t_{i j}$ are defined by the ratio of $r_{i j}$ to $r_{i m}$, such that $t_{i j}=\frac{r_{i j}}{r_{i m}}$.

The complexity of the overall transmission system can be seen in Figure 6, where the transmission relative to the fourth DoF (elbow joint) has been shown.

\subsection{Special components}

The mechanical design included some special components that were developed at PERCRO since no off-the-shelf components were found with the requested performance. Custom-designed components can not only match the required performance but can also be designed to be highly integrated with the remaining mechanical parts. The following components were developed: an open circular guide, an integrated planetary reduction gear and an integrated motor-group.

\subsubsection{Circular guide}

The circular guide (Figure 7) (Salsedo et al. 2001) is a special component specifically developed for the implementation of the rotational joint of the L-Exos. Since joint 3 is aligned with the axis of rotation of the human arm, a remote centre of rotation was needed. The solution of adopting a closed circular bearing was replaced with an open circular one, based on recirculating ball bearings technology. This innovative solution allows the user to don and doff the exoskeleton easily with no need to insert the arm through a closed-ring aperture or perform other uncomfortable maneuvers.

With respect to the closed bearings, the circular guide presents thae following two main advantages:

- Higher achievable mobility of the shoulder during the abduction-adduction movement due to the elimination

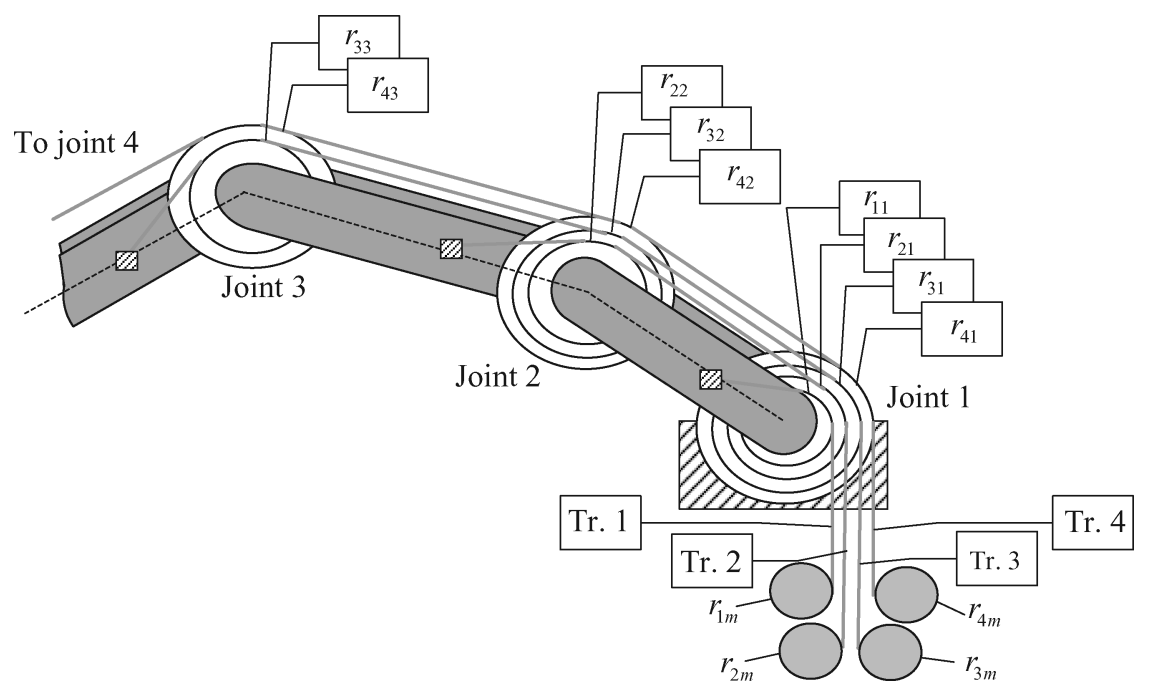

Figure 5. Diagram of the adopted routing for the four tendon transmissions. 
Table 2. Indication of gear and tendon speed reduction factors.

\begin{tabular}{|c|c|c|c|}
\hline \multicolumn{2}{|c|}{ Tendon reduction ratio } & \multicolumn{2}{|c|}{ Gear reduction ratio } \\
\hline$t_{i j}$ & Value & $g_{i i}$ & Value \\
\hline$t_{11}, t_{22}, t_{44}$ & 3.78 & $g_{11}$ & 6 \\
\hline$t_{21}, t_{22}, t_{23}$ & 2.78 & $g_{22}$ & 6 \\
\hline$t_{32}, t_{33}$ & 4.17 & $g_{33}$ & 1 \\
\hline$t_{33}, t_{43}$ & 13.11 & $\begin{array}{l}g_{44} \\
g_{44}\end{array}$ & 4 \\
\hline
\end{tabular}

of the internal bulk of component that limits the approaching of user's arm to his trunk.

- Simple ingress/egress.

The circular guide is mainly composed of a fixed part with a open circular geometry (railway) and a mobile part (cursor). The design of the component was carried out with the target of minimising the weight keeping unchanged the stiffness performances required for the particular application.

\subsubsection{The joint-integrated planetary reduction gear}

The reduction gear is the last element of the actuation system and is located on the joint axis. The primary target that was addressed during the design of the gearhead unit was the reduction of weight and volume with respect to standard commercial reduction gears. To fully pursue this target, a high level of integration with the axis joint was addressed (see Figure 8).

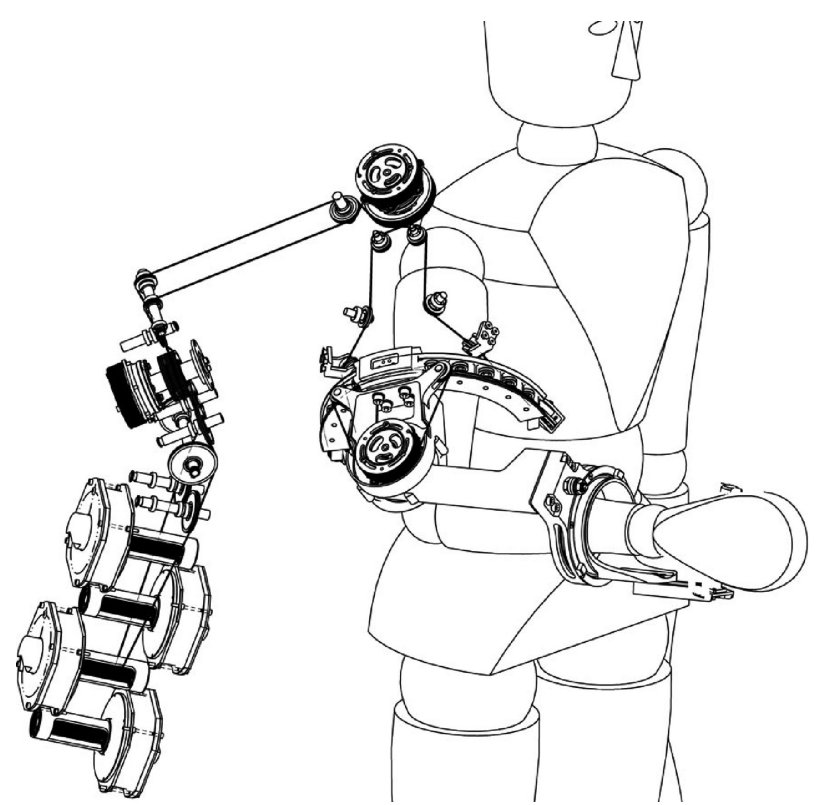

Figure 6. The complex routing of transmission of L-Exos is shown for the fourth transmission.

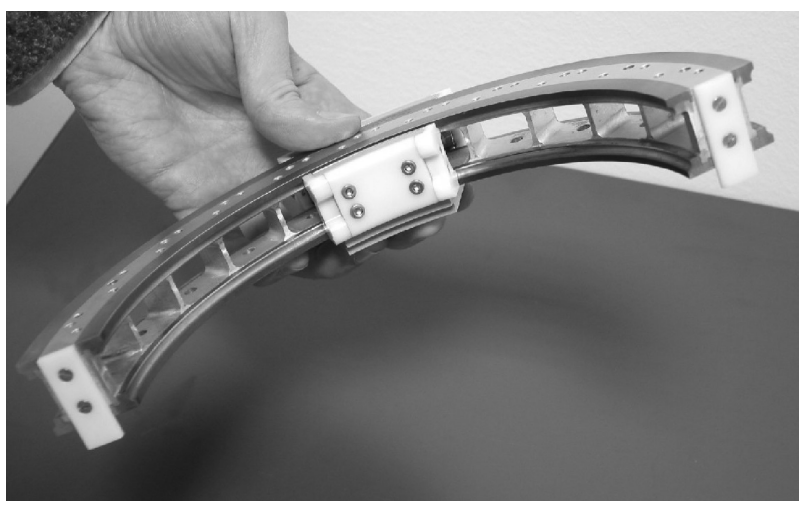

Figure 7. The open circular guide.

The number of parts was reduced by requiring gearhead to accomplish multiple functions, aside of being a speed reduction unit: the input shaft serves also as driven pulley of the transmission, while the output shaft is also the axle of each joint (see Figure 9). The interposition of the reduction gearhead between the tendon transmissions and the driven joint has also led to an enhancement of the stiffness of the system by reducing the tension of the steel cables composing the transmissions.

The outstanding lightness is achieved partially by means of the aforementioned integration, but mostly by means of a design effort that allowed us to use light alloy (aluminum alloy) instead of steel. The only parts of steel, apart from bolts and bearings, are the standard gears. A great effort was also carried out to maintain the backlash introduced by the gears within a tolerable value range. A lower range would imply a dramatic increase in the friction factor.

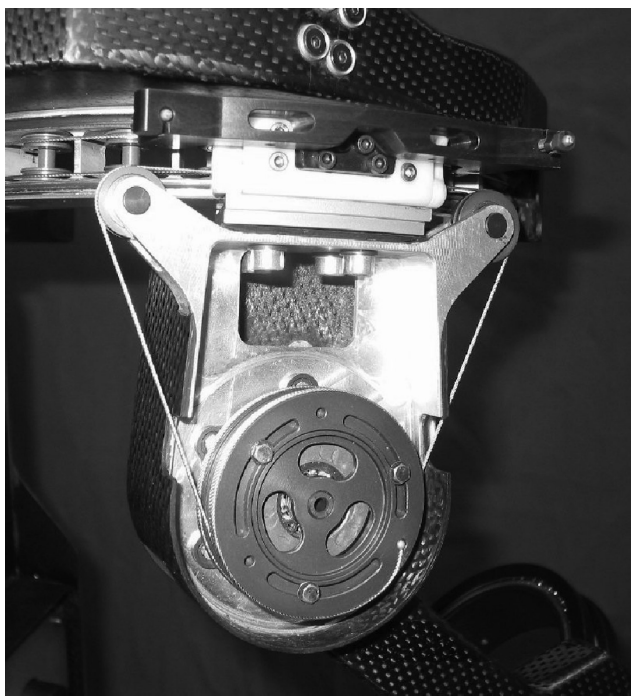

Figure 8. The integrated reduction gear assembled in the LEXOS joint. 


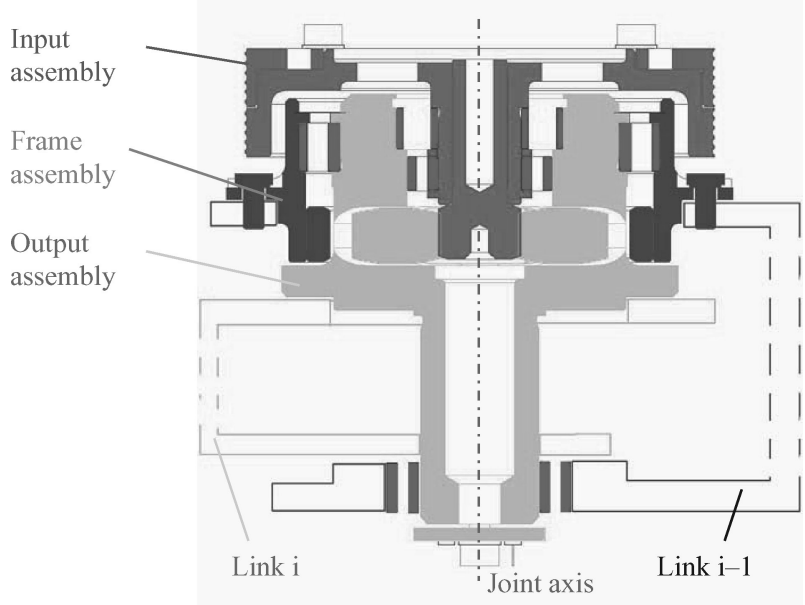

Figure 9. Cross-section of the integrated reduction gear.

\subsubsection{Motor group}

The four DoF are actuated by four identical motor groups. Each motor group is equipped with a frameless DC permanent magnet torque motor and a high-resolution optical encoder, as shown in Figure 10. Each motor can exert a maximum continuous torque of $2 \mathrm{Nm}$ and a peak one of $3.7 \mathrm{Nm}$. In the motor group, the driving pulley of the tendon drive is also included and the rotor of the motor is directly bonded on the driving pulley at its end side. The driving pulley is supported by two ball bearings located in correspondence of the two walls of the grounded link (link 0 ). The housing of the DC motor is cantilevered. All the structural parts of the motor group are made of lightweight alloy.

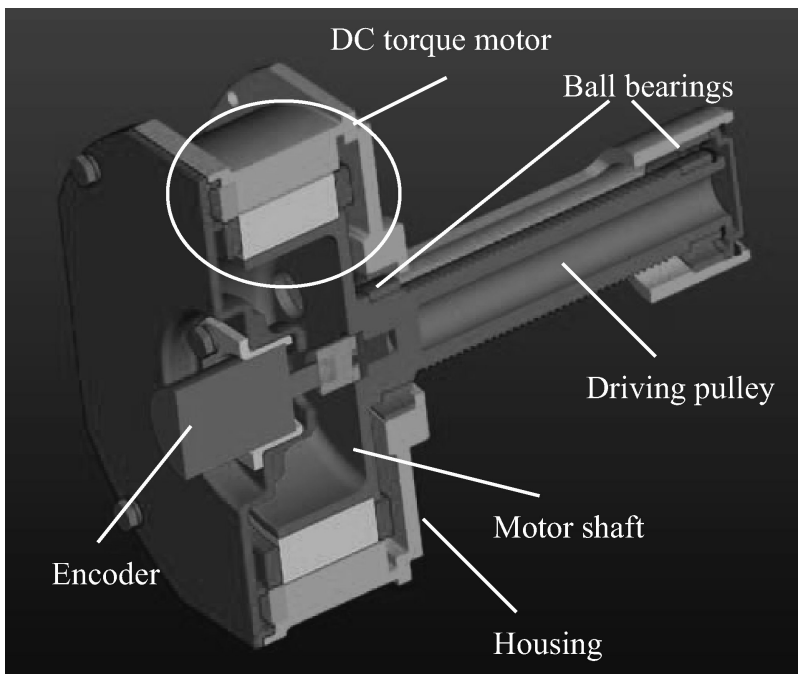

Figure 10. Scheme of the motorisation group.

\subsection{Performance of the integrated system}

The L-Exos can attain very remarkable performance that can be summarised as follows:

Payload: $\quad 50 \mathrm{~N}$ continuous, $100 \mathrm{~N}$ peak force.

Backlash: $\quad 10 \mathrm{~mm}$ at the end effector.

Stiffness: $\quad$ Estimated $3 \mathrm{~N} / \mathrm{mm}$, measured $2 \mathrm{~N} / \mathrm{mm}$.

Workspace: Approximately $70 \%$ of human arm.

The L-Exos has a weight of $11 \mathrm{~kg}$, of which approximately 6 $\mathrm{kg}$ is distributed on link 0 , i.e. the fixed part, and mostly due to the mass of the four motor groups. This means the L-Exos achieves the desirable very low value of weight/payload ratio of almost $1(100 \mathrm{~N}$ vs. $11 \mathrm{~kg})$. The reported value of stiffness of $3 \mathrm{~N} / \mathrm{mm}$ represents the theoretical worst-case condition. The experimental measurements provided a good confirmation of this value, even if the perceived stiffness seems to be amplified by the backlash introduced by joint gearheads.

\section{Experimental set-up}

\subsection{An integrated set-up for upper-limb rehabilitation}

Wearability and usability are crucial factors when dealing with impaired users, i.e. the structure must be as open as possible, in order not to cause any major difficulty for the patient to wear it. The structure of the L-Exos is open, the wrist being the only closed joint, and can therefore be easily worn by post-stroke patients with the help of a therapist. An adjustable height support was built, and a chair was placed in front of the device support, in order to enable patients to be comfortably seated while performing the tasks, as shown in Figure 11. The distance of the handle from the elbow was made adjustable according to the patient's arm length by means of a sliding guide, then fixed by bolts and nuts during the operating conditions. After wearing the robotic device, the subject's elbow is kept attached to the robotic structure by means of an elastic belt. If necessary,

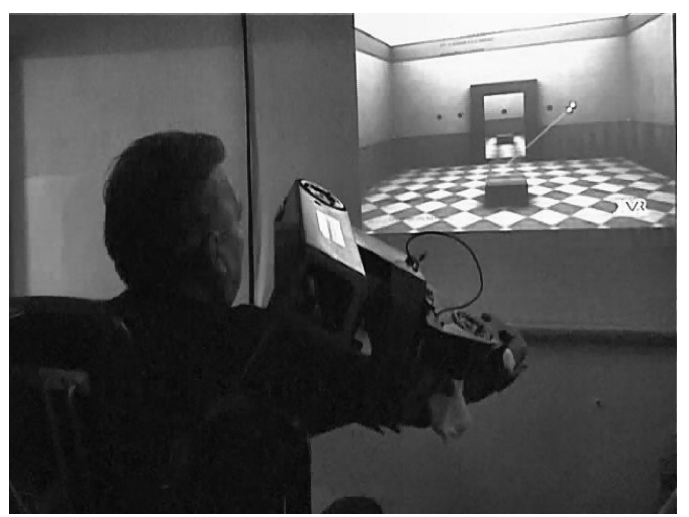

Figure 11. The exoskeleton in the configuration used for upper limb rehabilitation. 


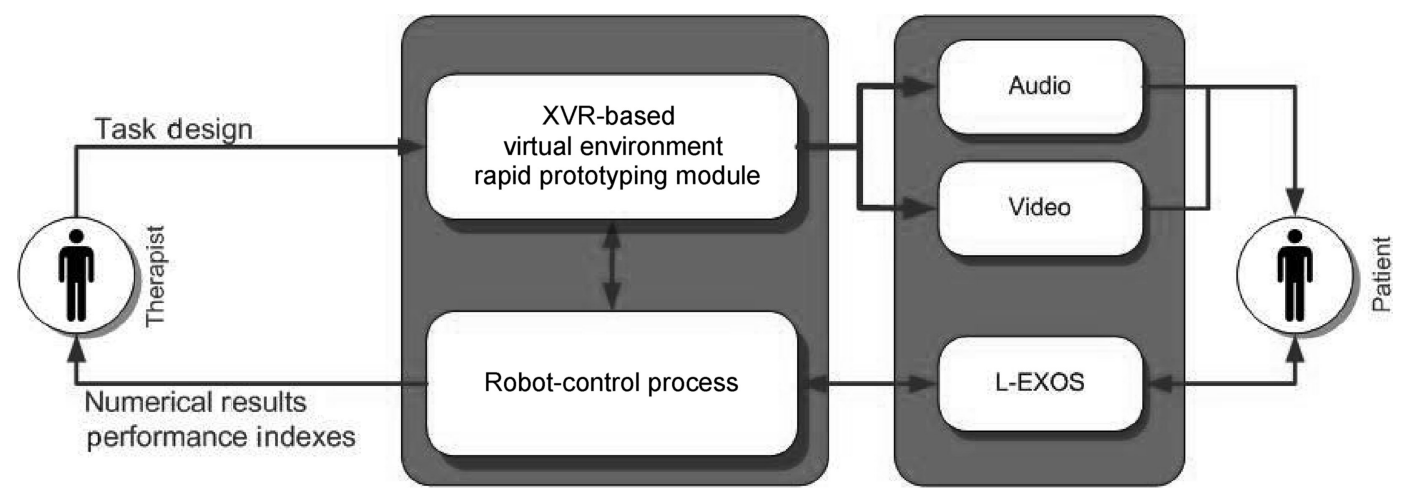

(a) Block diagram for robot-mediated therapy with L-Exos.

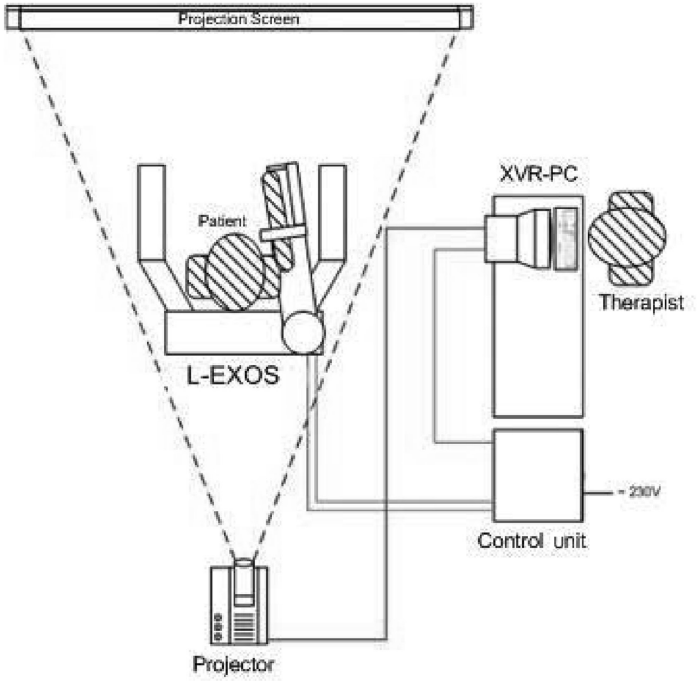

(b) System layout.

Figure 12. The experimental rehabilitation set-up.

the wrist may also be tightly attached to the device endeffector by means of a second elastic belt, which has been used for patients who are not able to fully control hand movements. Due to the fact that stroke patients tend to implement motor compensatory strategies to overcome their motor impairments, a third elastic belt can be employed in order to restrain the patient's torso compensatory movement. As a matter of fact, many patients tend to use postural movements when performing reaching tasks in the frontal plane, in order to reduce the extension movement required to the impaired elbow. The L-Exos safety system was also addressed in the design phase. Although the device has a wide workspace, no self-collisions with the body of the patient are possible due to mechanical stops that limit the workspace of the system. Software saturations and a redundant electric and electronics safety system were implemented in order to make the device fail-safe even in case of sudden power loss.

\subsection{Integration with $V R$}

The L-Exos device was integrated with a projector used to display on a wide screen placed in front of the patient with different virtual scenarios in which to perform rehabilitation exercises. The block diagram in Figure 12(a) represents the main components of the rehabilitation set-up. The patient, who interacts with the L-Exos system, is previously instructed about each exercise structure and receives both visual and acoustic feedbacks. He/she sees an avatar representing his/her movements in the virtual environment, and is able to recognise whether or not he/she is performing the correct task. Further, visual or acoustical feedback may help patients in understanding the exact starting and ending time instants for each exercise. Such feedback could also be interpreted as biofeedback to enhance patient's awareness of their performance for the required task. The therapist may change some of the task parameters according to the real-time analysis provided by the control unit. In particular, 


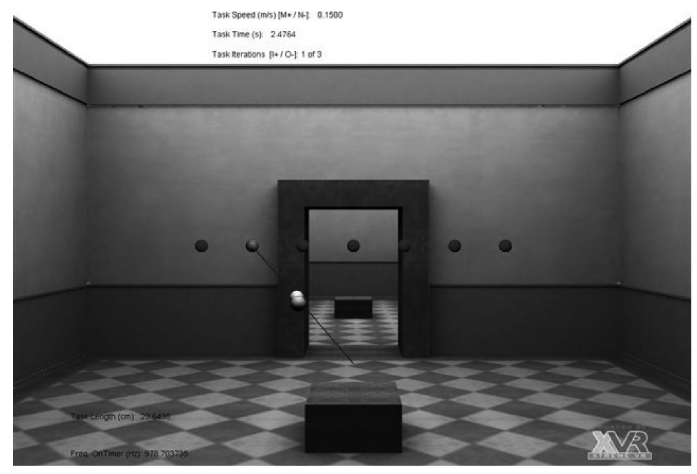

(a) The visualised virtual scenario.

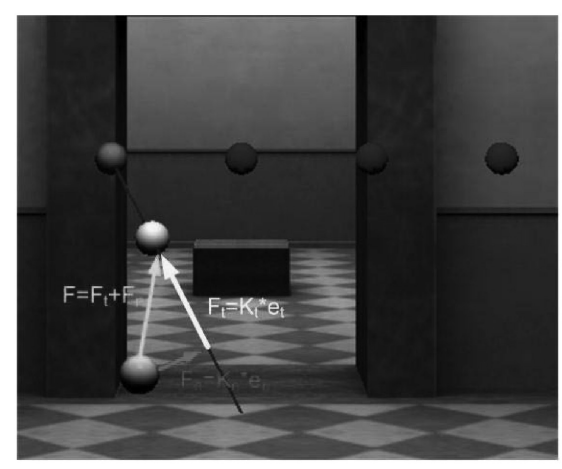

(b) The implemented impedance controller.

Figure 13. Reaching task application.

the therapist can modulate each therapy exercise difficulty level by interacting with a graphical user interface (GUI) on the control PC. The system can provide a feedback to the therapist by providing an instantaneous report of major numerical results and performance metrics for the proposed exercises.

The general layout of the final system is shown in Figure 12(b). The patient sits on the chair wearing the exoskeleton and performing the exercise, which is projected onto the screen. The therapist sits besides the patient, while adjusting significant parameters of each proposed exercise by a PC console.

\section{Development of an application for rehabilitation of reaching}

A virtual rehabilitation scenario was specifically developed using the XVR Development Studio (Ruffaldi et al. 2006) focused on the reaching movement. Reaching represents a fundamental activity of everyday life and has a high functional value. In this application it is possible to assist the patient in executing the required task, so that a passive movement of the upper limb occurs if the patient is not able to complete the task. During the execution, the following motor facilitations can be provided to the patient that can be adapted according to the stage of the therapy and gravity of motor impairment:

(1) Active limb support against gravitational load: This strategy leads to an increase of the workspace, reducing the associated abnormal shoulder/elbow muscle coactivation and joint torque coupling patterns in both static and dynamic tasks (Ellis et al. 2007). The level of upper limb weight compensation can be adjusted according to the specific requirements of each patient.

(2) Amplification of movement: In this way it is possible to put each patient in the conditions of executing the required task.
The scenario is composed of a virtual room, where different fixed targets are displayed to the patient as gray spheres disposed on a horizontal row, as shown in Figure 13(a). The position of the hand of the patient is shown as a green sphere, which is moved according to the L-Exos end-effector movements. According to the protocol specifications each target is successively selected and thus 'activated', i.e. it becomes red and a straight blue line connecting the starting position with the final target to be reached is displayed on the screen. The starting position of the task has been chosen as a rest position of the arm, with the elbow flexed at $90^{\circ}$. In this position, the exoskeleton provides the support for the weight of the arm so that the patient can comfortably lean his arm on the exoskeleton. After an acoustic signal indicating the start of the exercise, the patient is asked to keep the green sphere as close as possible to a yellow marker that moves along the line connecting the start and end points. The curvilinear coordinate of the marker is computed according to a minimum jerk model (Reinkensmeyer et al. 2000), i.e. a sigmoid-like shape which is approximated by a fifth degree polynomial with a bell-shaped displacement profile (see Figure 14). The patient is instructed to keep the green sphere as near as possible to the moving yellow sphere. The yellow marker reaches the target with zero velocity, and comes back on the blue line towards the initial position. The therapist can set the maximum speed of the task and change the number and position of the fixed targets that should be reached by the patient (both in terms of target height and depth within the virtual room).

\subsection{Detailed description}

The virtual scenario used for this task contains seven spheres in a horizontal row, on a virtual plane about $15 \mathrm{~cm}$ in front of the patient. Targets are initially idle and they are activated in sequence (i.e. the active target is displayed as a bright red sphere). Subjects are required to move their 


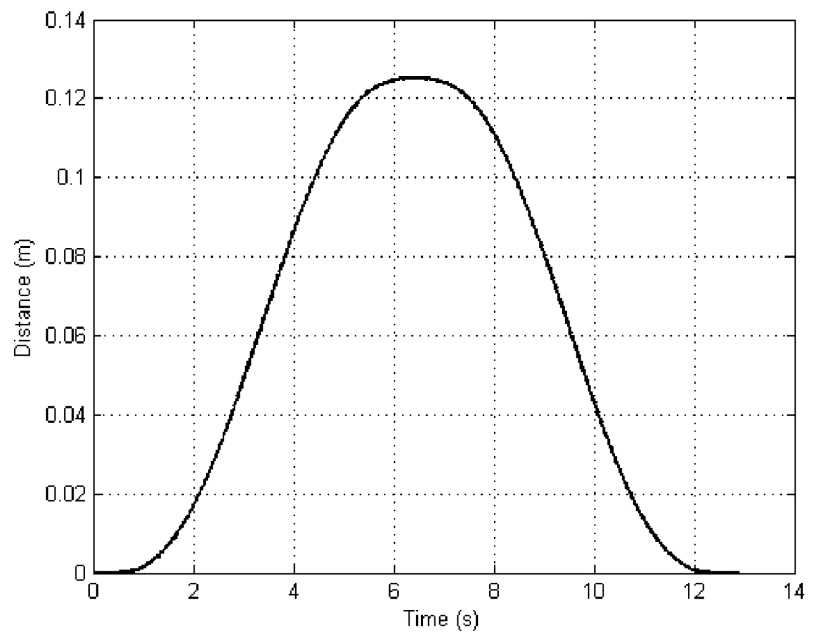

Figure 14. Ideal curvilinear coordinate along the blue line in the reaching task application.

hands towards the active target and then back to the initial position. Three different height levels (standard values: $h_{1}=0.01 \mathrm{~m}, h_{2}=0.12 \mathrm{~m}, h_{3}=0.18 \mathrm{~m}$, but they may be varied according to patient's needs and motor capabilities) have been employed for the sphere row for each patient in each therapy session. Task velocity can be set at two levels (standard values: $v_{1}=0.15 \mathrm{~m} / \mathrm{s}, v_{2}=0.20 \mathrm{~m} / \mathrm{s}$, but they may be varied according to patient's needs and motor capabilities). A two-second pause follows each complete reaching movement (i.e. forward and backward movement). The patient is required to perform three series of seven movements for each height level and for each velocity level, i.e. a total of seven (total number of spheres to be reached at each height level) $\times$ three (number of times each target at a certain height has to be reached at the same velocity level) $x$ three (different height levels) $\times$ two (different velocity levels) $=126$ forward and backward movements for each rehabilitation session. The duration of this exercise is about 30 minutes.

\subsection{Control}

An impedance control was adopted to leave the patient the possibility to actively conduct the task and being passively guided by the robot only when he/she is unable to complete the reaching task. A driving force is applied longitudinally to the desired trajectory, guiding the patient to the correct execution of the required movement and a constraint force is applied in the transversal direction to the motion to reduce the committed error. Two concurrent impedance controls acting along tangential and orthogonal directions to the trajectory were used to compute the desired force $\mathbf{F}$ at the end-effector, by projecting the Cartesian position error $\mathbf{e}$

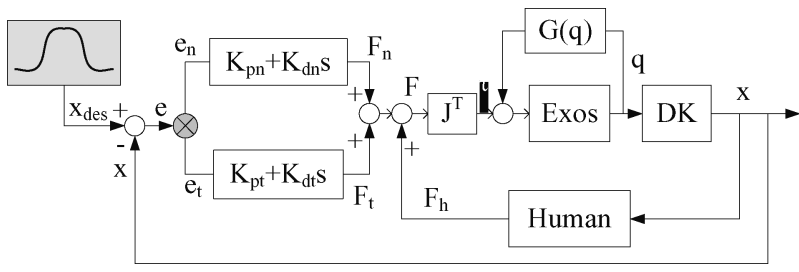

Figure 15. The impedance control scheme of the device in the reaching task.

along the two orthogonal directions $\mathbf{e}_{\mathbf{n}}$ and $\mathbf{e}_{\mathbf{t}}$ as

$$
\mathbf{F}=\mathbf{F}_{\mathbf{n}}+\mathbf{F}_{\mathbf{t}}=\left(K_{p n}+\mathrm{s} K_{d n}\right) \mathbf{e}_{\mathbf{n}}+\left(K_{p t}+\mathrm{s} K_{d t}\right) \mathbf{e}_{\mathbf{t}}
$$

This is illustrated in the control diagram of Figure 15 where the following notation is assumed:

$\mathbf{e}_{\mathbf{t}} \quad$ The tangential position error.

$\mathbf{e}_{\mathbf{n}} \quad$ The normal position error.

F The desired force applied on the operator's hand.

$\tau \quad$ The vector of the joint torques.

$J \quad$ The Jacobian.

q Joint position vector.

$\mathbf{x}_{\text {des }} \quad$ Target position of the end-effector.

$\mathbf{x} \quad$ Actual position of the end-effector.

e Position error: $\mathbf{x}_{\text {des }}-\mathbf{x}$.

$\mathbf{F}_{\mathbf{h}} \quad$ Force applied by the human operator.

$G(\mathbf{q}) \quad$ Gravity torques.

DK Direct kinematics module.

$K_{p n} \quad$ Control stiffness orthogonal to the trajectory: $1200 \mathrm{~N} / \mathrm{m}$.

$K_{p t} \quad$ Control stiffness tangential to the trajectory: $500 \mathrm{~N} / \mathrm{m}$.

Figure 16 shows the Cartesian position components (solid lines - patient) compared to the desired components

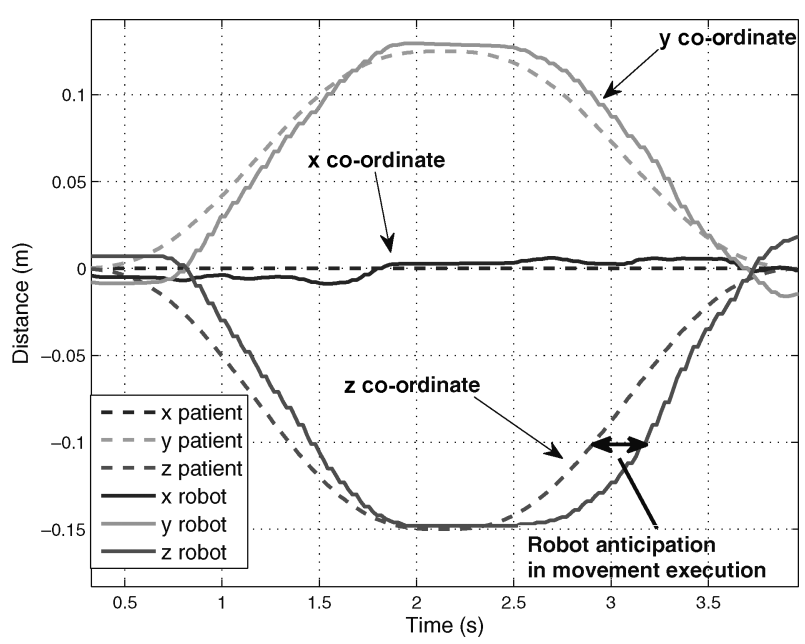

Figure 16. Patient performance (velocity $v_{3}=15 \mathrm{~cm} / \mathrm{s}$ ). 


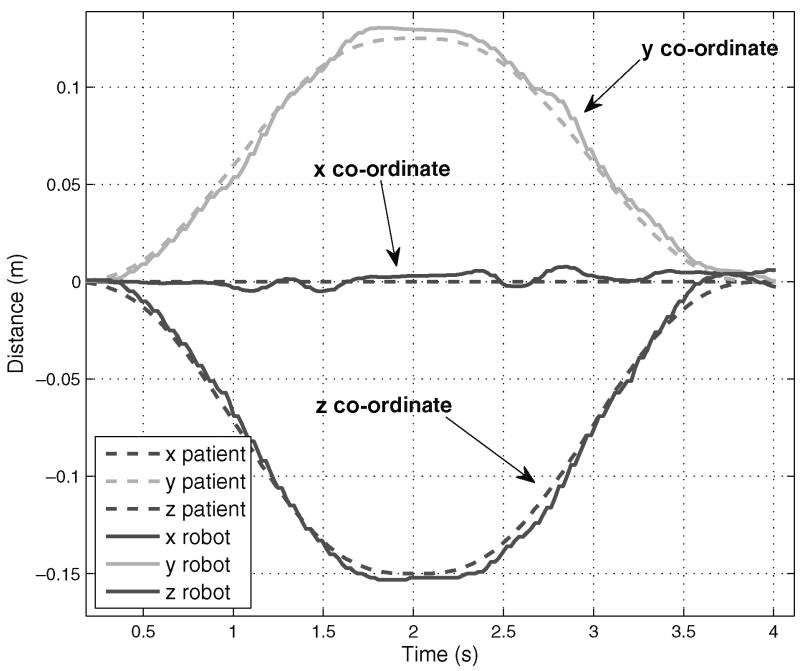

Figure 17. Healthy subject performance (velocity $v_{1}=5 \mathrm{~cm} / \mathrm{s}$ ).

(dashed lines - robot) of movement for one reaching task performed by one patient (60-year-old woman who suffered a meningioma in the left hemisphere of the brain) at the highest speed. The task was performed in the frontal plane with the $z$ axis directed opposed to the gravity direction and the $y$ axis along the frontal direction, so that the $x$ coordinate was always kept to zero.

The patient followed quite correctly the trajectory but with a delay between his/her hand and robot position. This indicates that the patient allows the device to drag his/her hand, being unable to actively accomplish the requested task. This can be better pointed out by comparing the patient's performance with one healthy volunteer, as shown in Figure 17 at a lower velocity. The healthy subject is able to actively follow the task and no significant delay is present between the target and the hand position.

The above results can be also analysed by observing the behaviour of the cumulative position error over time, both for the patient and for an healthy volunteer. Figure 18 compares two reaching tasks executed in the frontal plane. The plot outlines that the healthy subject presents a constant error rate, with the cumulative error increasing linearly over time. On the contrary, the patient presents an error rate composed of three segments with different slopes. The first and third segment have a higher slope and represent the reaching in the forward and backward directions, where a higher slope indicates a higher average error than the healthy subject. The error decreases only in the second segment, which represents the inversion point, where the direction of motion is reversed and the velocity is almost null.

From the analysis of Figure 18 it appears how the cumulative error curves can be used to describe the task performance of the patient during the reaching task, as they can significantly differentiate the performance of an healthy subject with the one of a motor-impaired subject.

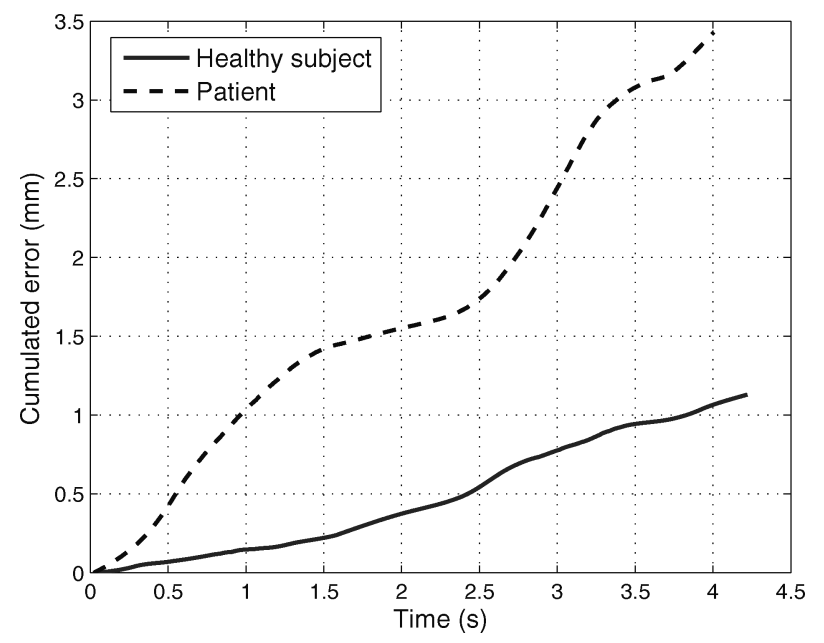

Figure 18. Cumulative position error during task execution $\left(v_{3}=\right.$ $15 \mathrm{~cm} / \mathrm{s})$.

\subsection{Evaluation on a group of eight patients}

A group of eight post-stroke chronic patients were enrolled in a therapy protocol, consisting of three one-hour rehabilitation sessions per week for a total of six weeks (i.e. 18 therapy sessions). We compared the performance of patients before and after therapy by computing, according to Figure 18, the maximum cumulative error performed by each patient in each reaching exercise, over different sessions.

Figure 19 shows the cumulative error-fitting curves for one selected target divided per patient per session, assuming the convention that red curves represent the first session, while green curves represent the last one. The analysis of the maximum performed error was restricted to the reaching tasks where the patient was asked to reach a central target located in the frontal plane, considering respectively the first and last four sessions of therapy for estimating the performance at the start and at the end of the therapy. Considering that each session was composed of three reaching tasks to a single target, a total of 12 executed reaching movements were evaluated for each patient.

Table 3. Quantitative results: mean maximum cumulative error \pm standard deviation.

\begin{tabular}{lrrlrr}
\hline Patient & \multicolumn{1}{c}{ Pre } & \multicolumn{1}{c}{ Post } & Signif. & $t$-stat & df \\
\hline 1 & $9.17 \pm 1.24$ & $10.79 \pm 1.55$ & - & -3.512 & 11 \\
2 & $15.88 \pm 7.19$ & $9.48 \pm 1.92$ & $p<0.01$ & 2.729 & 11 \\
3 & $5.36 \pm 0.98$ & $4.32 \pm 1.10$ & $p<0.05$ & 2.063 & 11 \\
4 & $7.65 \pm 1.00$ & $5.66 \pm 0.94$ & $p<0.001$ & 5.180 & 11 \\
5 & $6.26 \pm 0.72$ & $5.73 \pm 0.97$ & $p<0.05$ & 2.050 & 11 \\
6 & $5.95 \pm 1.03$ & $4.10 \pm 0.92$ & $p<0.001$ & 3.983 & 11 \\
7 & $6.12 \pm 1.46$ & $7.36 \pm 1.61$ & - & -1.812 & 11 \\
8 & $12.55 \pm 1.00$ & $14.16 \pm 1.58$ & - & -2.804 & 11 \\
\hline
\end{tabular}




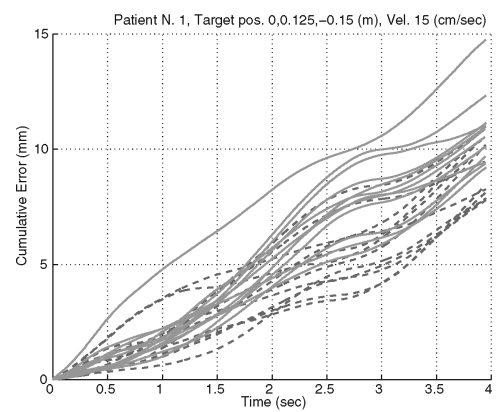

(a) Patient 1

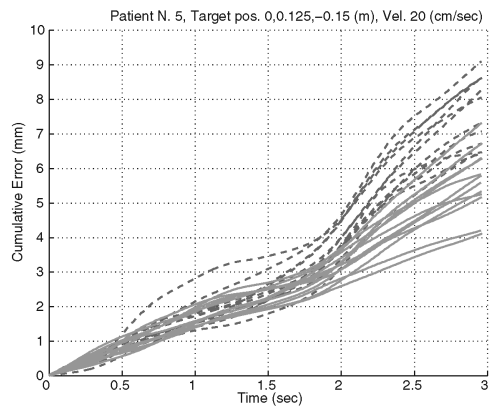

(d) Patient 4

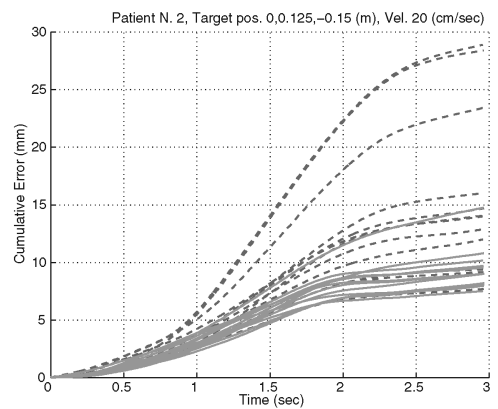

(b) Patient 2

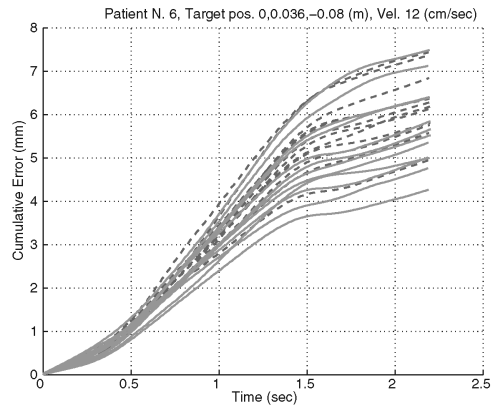

(e) Patient 5

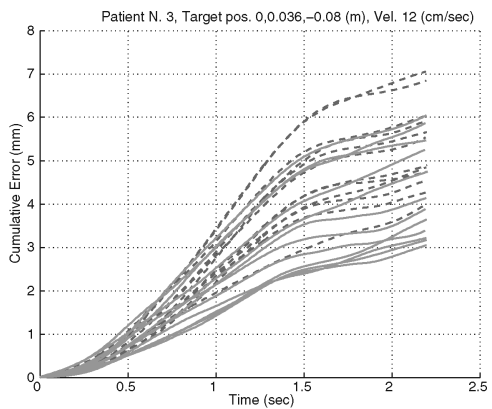

(c) Patient 3

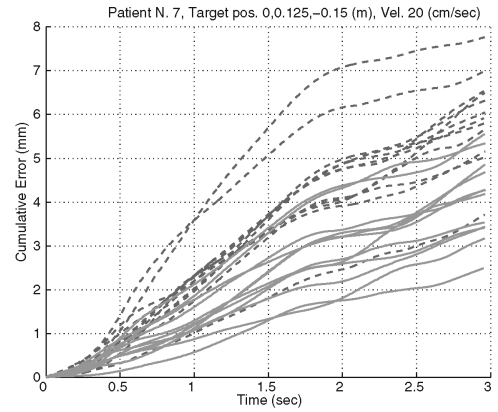

(f) Patient 6

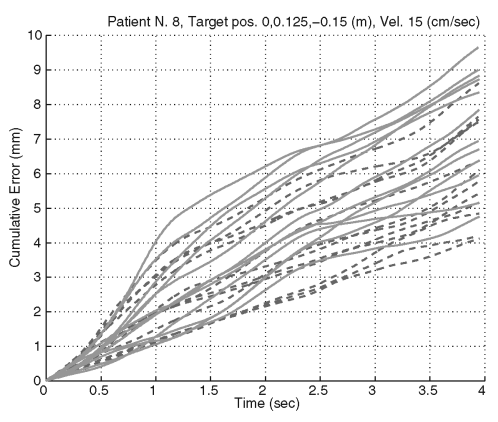

(g) Patient 7

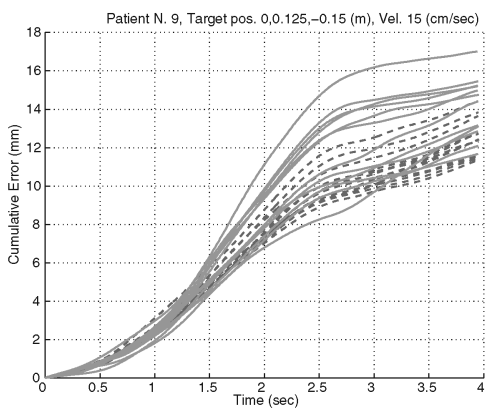

(h) Patient 8

Figure 19. Reaching task quantitative results. Continuous red and dashed green lines represent respectively the performance in the first and last four sessions executed during the therapy. This figure is available in colour online.

We observed an increment of performance in five out of eight patients ( $\sim 62.5 \%$ of the group) (single-tailed-paired $t$-test), as it can be shown by the data reported in detail in Table 3.

In the whole group, a single-tailed-paired $t$-test ( $t$-stat $=2.22, \mathrm{df}=95)$ showed a significant reduction of the performance error $(p<0.02)$ in the two conditions after (7.70 \pm 3.56$)$ vs. before $(8.61 \pm 4.41)$ therapy.

An interpretation of performance can be provided based on the analysis of the modification of the cumulative error curve before and after therapy in the following manner. For patients $2-6$ a typical improvement pattern is noticeable. The patients constantly improve their performance in the exercise, leading to a significant decrease in the final cu- mulative error for a given target. Moreover, a reducing of the mean slope of the central segment of the fitting curve is present, indicating a higher ability to maintain a lower average error throughout the task. No significant improvements can be observed in patients 1,7 and 8 .

\section{Conclusions and future work}

In this paper we have presented the mechanical design of the L-Exos, an upper-limb exoskeleton for force feedback in virtual environments. The L-Exos system was integrated in an experimental set-up for robotic-assisted neurorehabilitation in virtual reality and evaluated on a group of eight chronic stroke patients in the execution of 
robotic-assisted reaching. We believe that robotic-assisted therapy can bring great benefits to patients in terms of recovery of upper limb function, especially if the rehabilitation is carried out with spatial tasks involving the full mobility of patient's arm. This has been preliminarily demonstrated in this study through the definition of a suitable index of performance which allows to compare the performance of patients in the reaching task during therapy. As a future plan the authors plan to design a new version of the arm exoskeleton that can also include direct torque joint measurement and so selective control of arm joints.

\section{Acknowledgements}

This work was partially funded under a research grant by the Fondazione Monte Paschi Siena and by the IP SKILLS research project, funded by the European Commission. The authors acknowledge the contributions of Fabrizio Rocchi, Alberto Montagner and Luigi Borelli to this work.

\section{References}

Avizzano CA, Bergamasco M. 1999. Technological aids for the treatment of tremor. Proceedings of the Sixth International Conference on Rehabilitation Robotics (ICORR). Palo Alto, CA, USA.

Bergamasco M. 1996. Force replication to the human operator: the development of arm and hand exoskeletons as haptic interfaces. In: G. Giralt and G. Hirzinger, Robotics Research, The 7th International Symposium, p. 173-182, Springer-Verlag, London, 1996.

Bergamasco M, Allotta B, Bosio L, Ferretti L, Parrini G, Prisco GM, Salsedo F, Sartini G. 1994. An arm exoskeleton system for teleoperation and virtual environments applications. In: IEEE International Conference On Robotics and Automation, p. 1449-1454.

Cardoso LS, Costa R.da, Piovesana A, Costa M, Penna L, Crispin AC, Carvalho J, Ferreira H, Lopes ML, Brandao G. et al. 2006. Using virtual environments for stroke rehabilitation. International Workshop on Virtual Rehabilitation, 2006 p. 1-5.

Carignan CR, Naylor MP, Roderick SN. 2008. Controlling shoulder impedance in a rehabilitation arm exoskeleton. ICRA 2008 IEEE International Conference on Robotics and Automation, 2008. p. 2453-2458.

Diller L. 2000. Post-stroke rehabilitation practice guidelines. In: International handbook of neuropsychological rehabilitation. Critical issues in neurorehabilitation. New York: Plenum; p. 167-182.

Ellis MD, Sukal T, DeMott T, Dewald J. 2007. ACT 3D exercise targets gravity-induced discoordination and improves reaching work area in individuals with stroke. ICORR 2007. IEEE 10th International Conference on Rehabilitation Robotics, 2007. p. 890-895.

Fasoli SE, Krebs HI, Stein J, Frontera WR, Hogan N. 2003. Effects of robotic therapy on motor impairment and recovery in chronic stroke. Arch Phys Med Rehabil. 84(4):477-82.

Frisoli A, Rocchi F, Marcheschi S, Dettori A, Salsedo F, Bergamasco M. 2005. A new force-feedback arm exoskeleton for haptic interaction in virtual environments. WHC 2005. First Joint Eurohaptics Conference and Symposium on Haptic Interfaces for Virtual Environment and Teleoperator Systems, 2005. p.195-201.

Gupta A, OMalley MK. 2006. Design of a haptic arm exoskeleton for training and rehabilitation. IEEE/ASME Transactions on Mechatronics, 11(3):280-289.
Jack D, Boian R, Merians AS, Tremaine M, Burdea GC, Adamovich SV, Recce M, Poizner H. 2001. Virtual realityenhanced stroke rehabilitation. IEEE Transactions on Neural Systems and Rehabilitation Engineering [see also IEEE Trans. on Rehabilitation Engineering], 9(3):308-318.

Jau BM. 1988. Anthropomorhic Exoskeleton dual arm/hand telerobot controller. IEEE International Workshop on Intelligent Robots, 1988. p. 715-718.

Kiguchi K, Iwami K, Yasuda M, Watanabe K, Fukuda T. 2003. An exoskeletal robot for human shoulder joint motion assist. IEEE/ASME Transactions on Mechatronics 8(1):125135.

Kiguchi K, Kariya S, Watanabe K, Izumi K, Fukuda T. 2001. An exoskeletal robot for human elbow motion supportsensor fusion, adaptation, and control. IEEE Transactions on Systema, man and cybernetics - Part B: Cybernetics. 31(3):353.

Krebs HI, Hogan N, Aisen ML, Volpe BT. 1998. Robot-aided neurorehabilitation. IEEE Transactions on Rehabilitation Engineering. [see also IEEE Trans. on Neural Systems and Rehabilitation], 6(1):75-87.

Lum PS, Burgar CG, Shor PC, Majmundar M, Van der Loos M. 2002. Robot-assisted movement training compared with conventional therapy techniques for the rehabilitation of upperlimb motor function after stroke. Arch Phys Med Rehabil. 83(7):952-959.

Nahvi A, Nelson DD, Hollerbach JM, Johnson DE. 1998. Haptic manipulation of virtual mechanisms from mechanical CAD designs. Paper presented at: Robotics and Automation 1998. Proceedings of 1998 IEEE International Conference on Robotics and Automation (ICRA'98), Leuven, Belgium.

Nef T, Riener R. 2005. ARMin-design of a novel arm rehabilitation robot. ICORR 2005. 9th International Conference on Rehabilitation Robotics, 2005. p. 57-60.

Perry JC, Rosen J, Burns S. 2007. Upper-limb powered exoskeleton design. IEEE/ASME Transactions on Mechatronics. 12(4):408-417, Aug.

Prange GB, Jannink MJ, Groothuis-Oudshoorn CG, Hermens HJ, Ijzerman MJ. 2006. Systematic review of the effect of robotaided therapy on recovery of the hemiparetic arm after stroke. $J$ Rehabil Res Dev. 43(2):171-184.

Reinkensmeyer DJ, Kahn LE, Averbuch M, McKenna-Cole A, Schmit BD, Rymer WZ. 2000. Understanding and treating arm movement impairment after chronic brain injury: progress with the ARM guide. J Rehabil Res Dev. 37(6):653662.

Riener R, Nef T, Colombo G. 2005. Robot-aided neurorehabilitation of the upper extremities. Med Biol Eng Comput. 43(1):210.

Ruffaldi E, Frisoli A, Bergamasco M, Gottlieb C, Tecchia F. 2006. A haptic toolkit for the development of immersive and webenabled games. Proceedings of the ACM Symposium on Virtual Reality Software and Technology, p. 320-323.

Salsedo F, Dettori A, Bergamasco M. 2001. Tendon-driven rotational joint for exoskeleton structure. World Patent WO2004058457, 2004-07-15.

Salsedo F, Dettori A, Frisoli A, Rocchi F, Bergamasco M, Franceschini M. 2002. Exoskeleton interface apparatus. World Patent WO2004058458, 2004-07-15.

Stewart JC, Yeh SC, Jung Y, Yoon H, Whitford M, Chen SY, Li L, McLaughlin M, Rizzo A, Winstein C.J. 2006. Pilot trial results from a virtual reality system designed to enhance recovery of skilled arm and hand movements after stroke. International Workshop on Virtual Rehabilitation, 2006. p. 18-23.

Tsagarakis NG, Caldwell DG. 2003. Development and control of a "soft-actuated" exoskeleton for use in physiotherapy and training. Autonomous Robots. 15(1):21-33. 

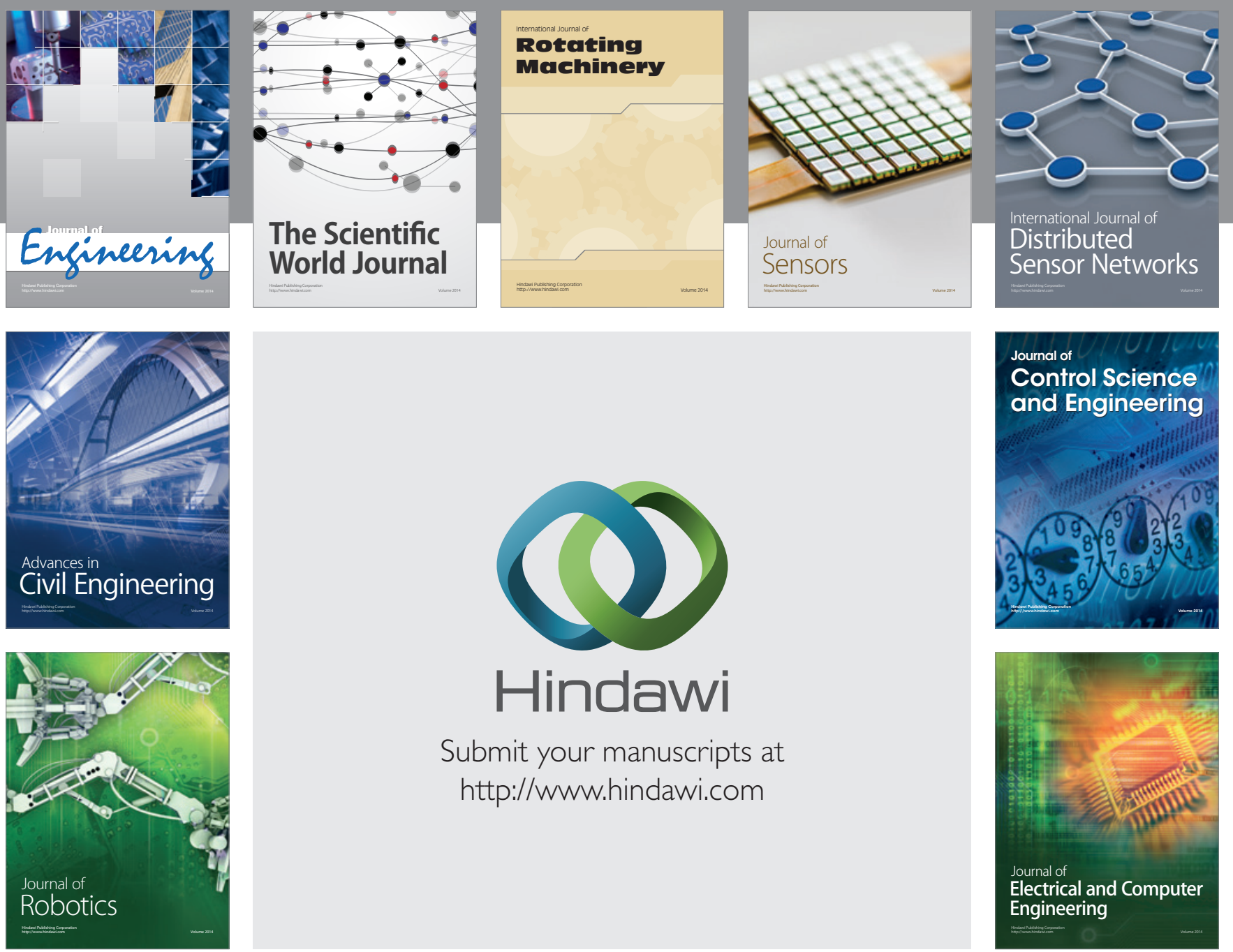

Submit your manuscripts at

http://www.hindawi.com
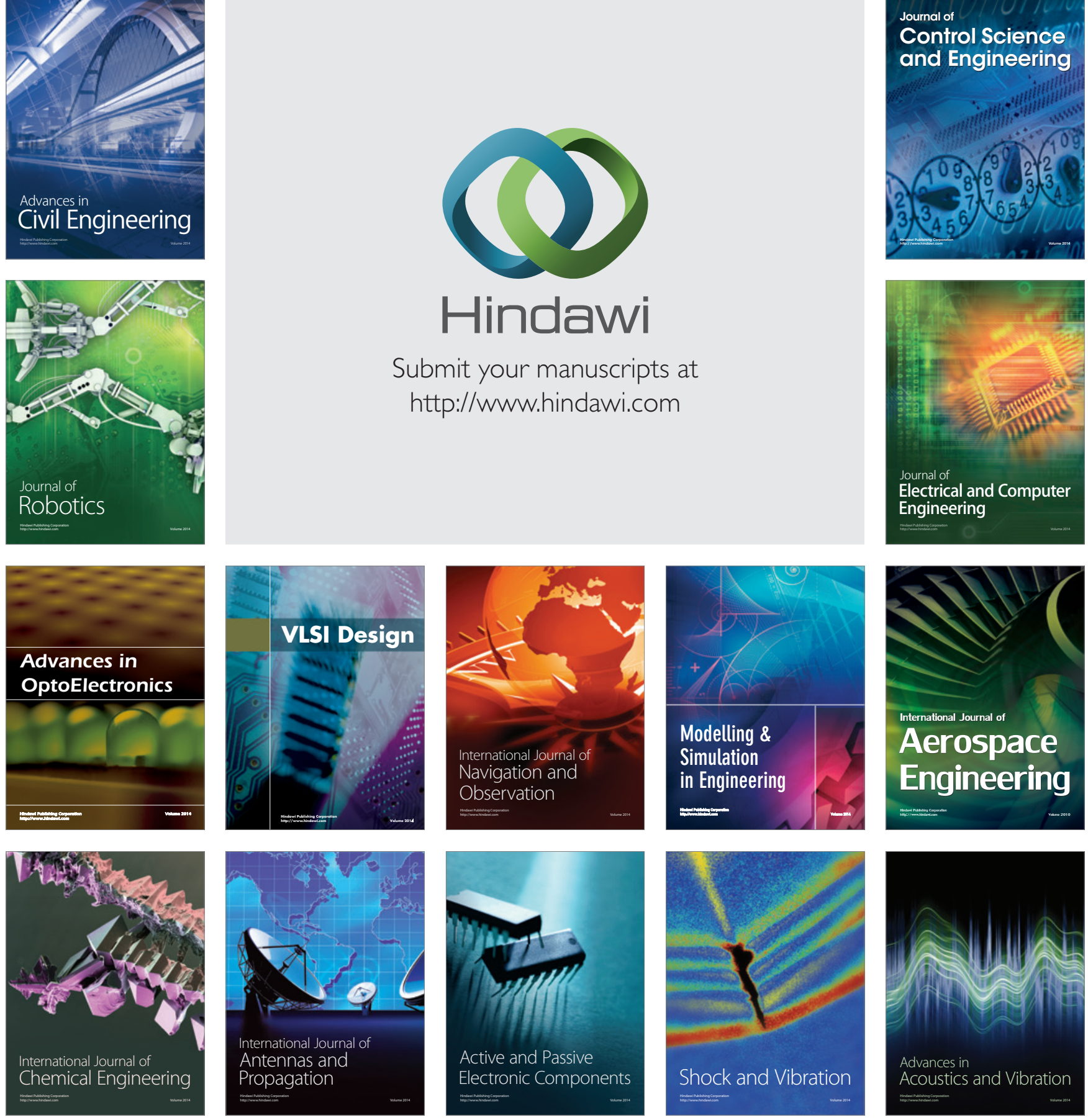